\title{
Renal epithelioid angiomyolipoma with epithelial cysts: demonstration of Melan A and HMB45 positivity in the cystic epithelial lining
}

\author{
José do Egypto Pereira Filho, MD ${ }^{\mathrm{a}}$, Daniele Meneses de Amorim ${ }^{\mathrm{b}}$, \\ Gloria Maria Maranhão Sweet, $\mathrm{MD}^{\mathrm{c}}$, Luiz Antonio Rodrigues de Freitas, $\mathrm{MD}, \mathrm{PhD}^{\mathrm{b}, \mathrm{c}, \mathrm{d}}$, \\ Paulo Roberto Fontes Athanazio, MD, MsC ${ }^{\mathrm{b}, \mathrm{c}}$, Daniel Abensur Athanazio, $\mathrm{MD}, \mathrm{PhD}^{\mathrm{b}, *}$ \\ ${ }^{\mathrm{a}}$ Hospital Aeroporto, Lauro de Freitas, Brazil \\ ${ }^{\mathrm{b}}$ Federal University of Bahia, Salvador, Brazil \\ ${ }^{\mathrm{c}}$ Imagepat Anatomic Pathology Laboratory, Salvador, Brazil \\ ${ }^{\mathrm{d}}$ Gonçalo Moniz Research Center, Oswaldo Cruz Foundation, Salvador, Bahia
}

\begin{abstract}
Renal angiomyolipoma (AML) may present as rare variants such as epithelioid and AML with epithelial cysts posing difficulties for the diagnosis to the surgical pathologist. We report a case of a 46-year-old male patient presenting a 5-cm solid tumor in the lower pole of the left kidney, with cystic changes at cut surface. The tumor exhibited $95 \%$ of epithelioid cells with atypical nuclei. A small focus of typical AML was observed. The immunoprofile of tumor cells was classical of AML including expression of melanocytic markers such as HMB45 and Melan A. We report the immunohistochemical study of the cystic component in an epithelioid AML. In contrast to the immunoreactivity reported in typical AML, the present case shows obvious expression of melanocytic markers in the cystic epithelial lining. This is strong evidence that these cysts are neoplastic and derived from AML, rather than entrapped native collecting duct epithelium.

(C) 2012 Elsevier Inc. All rights reserved.
\end{abstract}

\section{Introduction}

Angiomyolipoma (AML) is a benign mesenchymal tumour composed of a variable proportion of adipose tissue, spindle and epithelioid smooth muscle cells, and abnormal thick-walled blood vessels, accounting for $1 \%$ of all surgically removed renal neoplasms [1]. A minority of cases is related to tuberous sclerosis. Epithelioid AML (EAML) was recently recognized as a distinct clinicalpathologic entity, and it is defined as a potentially malignant mesenchymal neoplasm characterized by proliferation of predominantly epithelioid cells that is closely related to the triphasic (classic) AML [2]. Epithelioid AML shares with AML the characteristic HMB45 immunoreactivity of single

* Corresponding author. Universidade Federal da Bahia, Instituto de Ciências da Saúde. Av Reitor Miguel Calmon s/n, Vale do Canela 40110100 - Salvador, BA - Brasil. Tel.: +55(71) 3283889 Ramal: 41; fax: +55 71 32404194

E-mail address: daa@ufba.br (D.A. Athanazio). or small group of cells. Epithelioid AML also diffusely express CD68, CD34, and progesterone receptor (PR); exhibit a variable vimentin and smooth muscle actin expression; and focally express CD117, cytokeratins, and epithelial membrane antigen. The absence of S100 protein expression is an important feature to distinguish it from primary or metastatic melanomas [3].

Until 2009, 69 cases of EAML could be retrieved from English literature. A major concern regarding EAML is that its morphology, especially when lacking typical AML foci, may be easily misinterpreted as a renal cell carcinoma. Typical immunophenotypes HMB45+, S100-, cytokeratin AE1/AE3 - would identify EAML, but it would be a laborious and costly task to perform immunohistochemistry in all renal neoplasms in the surgical pathology practice [3]. Other rare variant of AML is the so-called AML with epithelial cysts that poses to the pathologist a wider range of differential diagnoses [4-6]. We report herein the immunohistochemical study of the cystic component in an EAML. In contrast to the immunoreactivity reported in typical AML, 
the present case shows obvious expression of melanocytic markers in the cystic epithelial lining. This is strong evidence that these cysts are neoplastic rather than entrapped native collecting duct epithelium.

\section{Case report}

A 46-year-old male patient underwent an abdominal ultrasonography as an complementary investigation for chronic gastritis. The ultrasonographic finding of an incidental $6.0-\mathrm{cm}$ ill-defined renal mass led the patient to seek assistance in a referral center of urologic surgery. The patient did not exhibit any signal or symptoms of the tuberous sclerosis complex.

The patient was submitted to a lower-pole partial left nephrectomy. At surgery, no other cysts were identified in the kidney. The specimen showed a tumor measuring $5 \mathrm{~cm}$ in its maximum diameter, protruded from the renal capsule. The cut surface exhibited yellowish solid and cystic zones (Fig. 1). The largest cystic space measured $3.0 \times 2.0 \mathrm{~cm}$. Cysts were filled by serous or hemorrhagic fluid. Microscopically, the tumor was well defined, composed by large epithelioid cells with abundant eosinophilic cytoplasm (Fig. 2A). Some cells exhibited clear or foamy cytoplasm. The nuclei of neoplastic cells were large and irregular, with prominent nucleoli (Fig. 2B). More than 95\% of all tumor areas showed this morphologic pattern. The neoplastic cells exhibited focal expression of HMB45 and diffuse and strong expression for Melan A (Fig. 2C, D). The cells also stained positive for vimentin, smooth muscle actin, and CD10. The tumor exhibited also a weak and focal expression of cytokeratin (AE1/AE3), while no expression of cytokeratin 7, c-KIT, estrogen receptor (ER), PR, and S-100 protein was detected. The proliferation marker Ki67 was expressed in less than $5 \%$ of tumor cells in solid areas and in epithelial lining of cysts. Table 1 details clones and manufacturer's

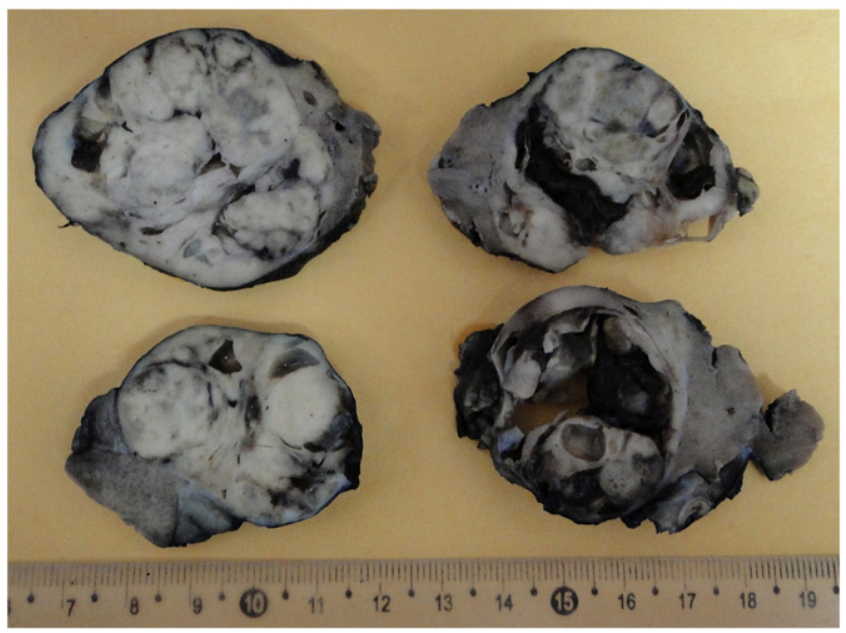

Fig. 1. Gross appearance: the cut surface of the solid-cystic tumor protruded from the renal capsule. information on the antibodies used. In a single focus, typical areas of smooth muscle and fat could be identified (Fig. 3A). In other areas, hyalinized blood vessels were seen (Fig. 3B). Mitotic count was lower than 1 per high-power field. Atypical mitoses were not detected. Areas of necrosis or giant cells were not observed.

The tumor exhibited cystic spaces of various sizes lined by cuboidal or hobnail cells. Many cysts were observed only at microscopical level. The epithelial lining of these cysts showed immunoreactivity for HMB45 (sparse positive cells) and Melan A (strong and diffuse pattern; Fig. 4).

The tumor had no evidence of extension to renal parenchyma, surgical margins, or perirenal fat.

\section{Discussion}

It has been suggested that some features may facilitate the pathologist to avoid a misdiagnosis of renal cell carcinoma when dealing with an EAML: presence of typical AML areas organized in single or multiple nodules; unusual location (such as perirenal adipose tissue and renal papilla); giant multinucleated cells with peripheral distribution of nuclei and voluminous, frothy cytoplasm; variegated microscopic appearances; extensive areas of plasmacytoid and/or rhabdoid cell change; and melanin deposition [3]. Except for a single focus of typical AML, the other criteria were absent in the present case.

A meta-analysis enrolling all 69 cases from English literature until 2009 described the most common features of EAML: mean age at diagnosis, $44 \pm 16$ years; female/male ratio, $3: 1$, and $26 \%$ associated with tuberous sclerosis. In at least 13 cases, the first diagnosis was renal cell carcinoma. At diagnosis, the tumors measured a mean of $10.0 \pm 6.0 \mathrm{~cm}$. Among patients with available follow-up, 38\% (16/48) showed malignant potential based on local aggressiveness (lymph node and adrenal involvement, or local recurrence, in 6 cases, with 3 deaths, or distant metastasis/multiorgan involvement in 10 cases, with 6 deaths) [3]. Importantly, all this information is from single case reports or small series, and the diagnosis of EAML did not follow any criterion of minimum epithelioid component.

In a large series of AML $(n=194)$, epithelioid areas could be identified in $15(8 \%)$ of all cases. Microscopic foci, epithelioid component, and epithelial cysts were all associated with tuberous sclerosis. In the case of epithelioid appearance, it was observed in $4(25 \%)$ of 16 cases with and $11(6 \%)$ of 179 cases without tuberous sclerosis. In this series, EAML was arbitrarily defined as AML with at least $10 \%$ of epithelioid component. Using the criteria of $100 \%$ epithelioid morphology, EAML is even rarer accounting for less than 1\% (2/194) of all AMLs. Because none of these 15 EAML cases exhibited malignant course (after a mean 5.1 years of follow-up), it was suggested that the previous inference of $30 \%$ malignant behavior of EAML could be explained by biased overestimation due to highly selectivity 

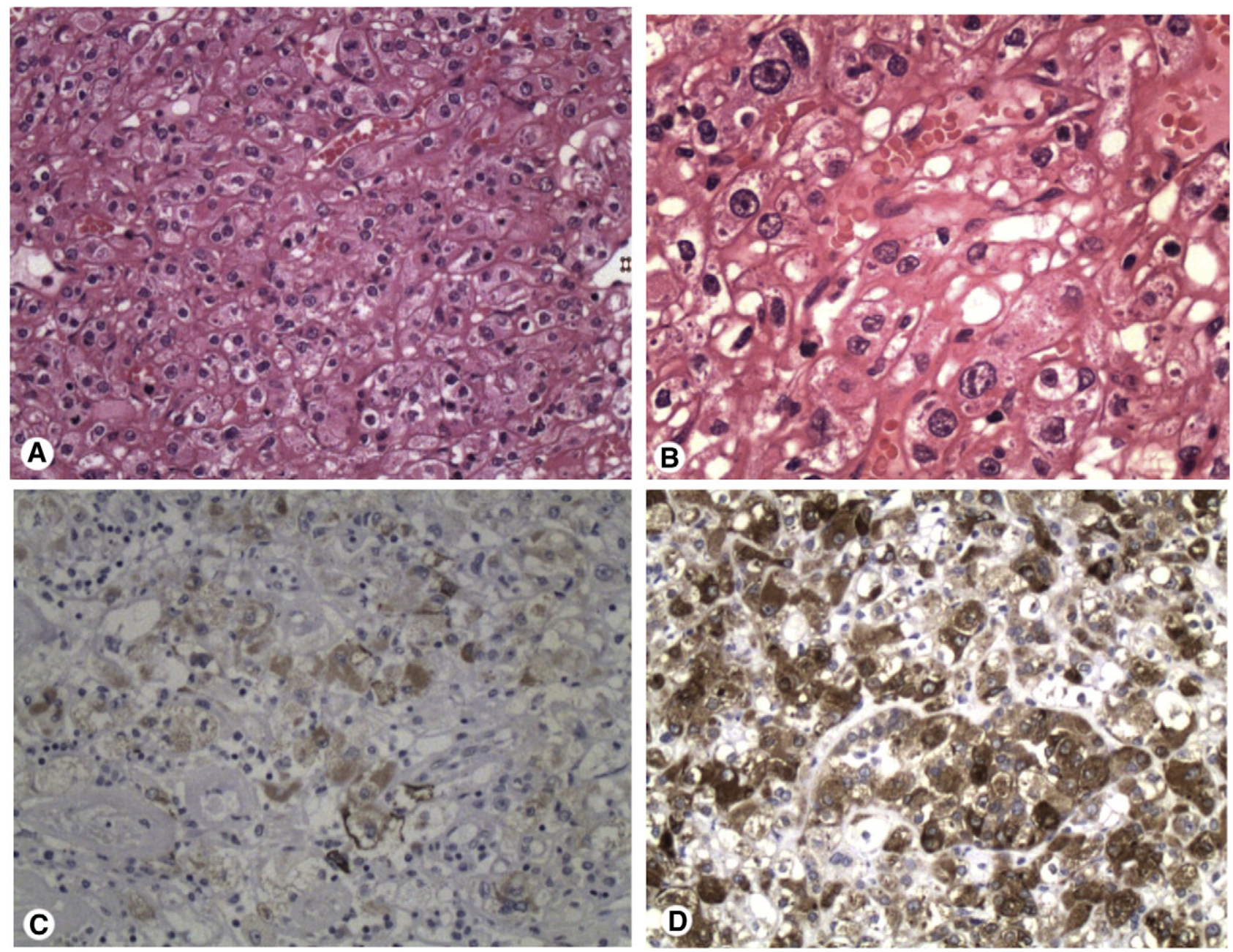

Fig. 2. Microscopic features of the EAML. (A) Predominant solid areas of epithelioid cells (hematoxylin and eosin, magnification $\times 200$ ). (B) Nuclear atypia of tumor epithelioid cells (hematoxylin and eosin, magnification $\times 400$ ). (C) Focal expression of HMB45 in epithelioid tumor cells (magnification $\times 200$ ). (D) Diffuse expression of Melan A in epithelioid tumor cells (magnification $\times 400$ ).

of case reports or small series [7]. The same group, however, reported later a series of 40 EAML with atypical cytology (now arbitrarily defining EAML with at least 5\% epithelioid

Table 1

Clones and manufacturers of the antibodies used in this case

\begin{tabular}{lll}
\hline Target antigen & Antibody clone & Manufacturer \\
\hline Cytokeratin & AE1/AE3 & DAKO \\
Cytokeratin 7 & OV-TL 12/30 & DAKO \\
CD10 & 56C6 & Vision BioSystem \\
CD117 (C-Kit) & Polyclonal & DAKO \\
ER & 1D5 & DAKO \\
Ki67 & Mib-1 & DAKO \\
Melanoma & HMB45 & DAKO \\
Melan A & A103 & DAKO \\
PR & PgR 636 & DAKO \\
S100 protein & Polyclonal & Zymed \\
Smooth muscle actin & 1A4 & DAKO \\
Vimentin & Vim 3B4 & DAKO
\end{tabular}

Abbreviations: DAKO, Glostrup, Denmark; Vision Biosystem, Norwell, MA, USA; Zymed, San Francisco, CA, USA. morphology). In this series, the following features of aggressive disease were listed: lymphovascular invasion $(n=3)$, renal vein invasion $(n=3)$, hilar involvement $(n=5)$, perinephric fat involvement $(\mathrm{n}=6)$, recurrence or metastasis $(n=9)$, and death $(n=4)$. The authors elaborated a predicted model of clinically malignant course (able to identify $78 \%$ of malignant and $100 \%$ of benign outcomes) based on the following morphologic criteria: $70 \%$ or more epithelioid cell component, 2 or more mitotic figures per 10 high-power fields, atypical mitotic figures, and necrosis [8]. In the present case, the only predictor of aggressiveness was the extensive (>95\%) epithelioid component.

In the present case, as in most cases of AML and EAML, the tumor was predominantly solid. A cystic variant of AML has been reported in 17 cases [4-6,9]. In 9 of those 16 previously reported cases, tumors were described as predominantly solid nodules with cystic component [4-6]. In cases with predominantly cystic morphology, the most challenging differential diagnosis would be mixed epithelial and stromal tumor, as well as multilocular cystic renal cell 

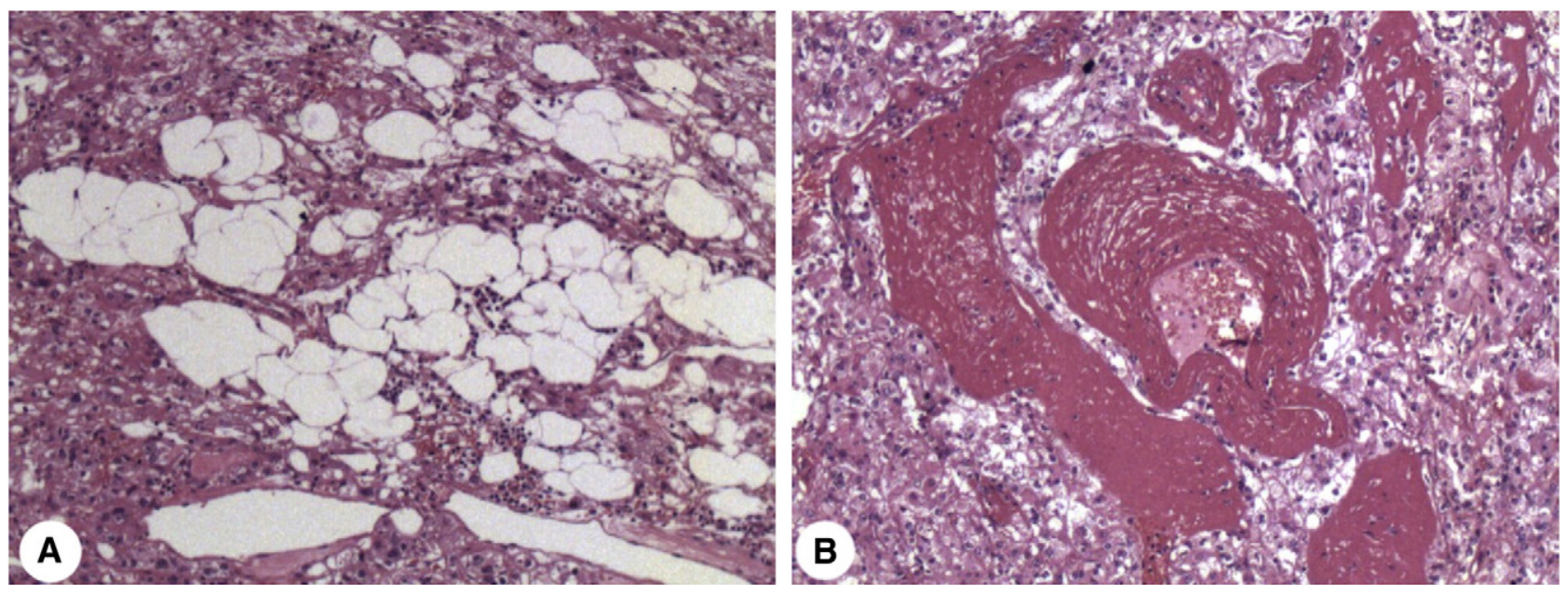

Fig. 3. Small zone of typical AML containing well-differentiated smooth muscle and fat (hematoxylin and eosin, magnification $\times 200$ ) and hyalinized blood vessels within the epithelioid solid areas (hematoxylin and eosin, magnification $\times 100$ ).

carcinoma [4]. In the largest series, only 2 of 11 cases were recognized as AMLs at first diagnosis [5]. The case reported herein were partially cystic, and the cystic spaces were lined by cuboidal or hobnail cells showing immunoreactivity for HMB45 and Melan A, which was not observed in previous reports of so-called AML with epithelial cysts $[4,6,9]$ or
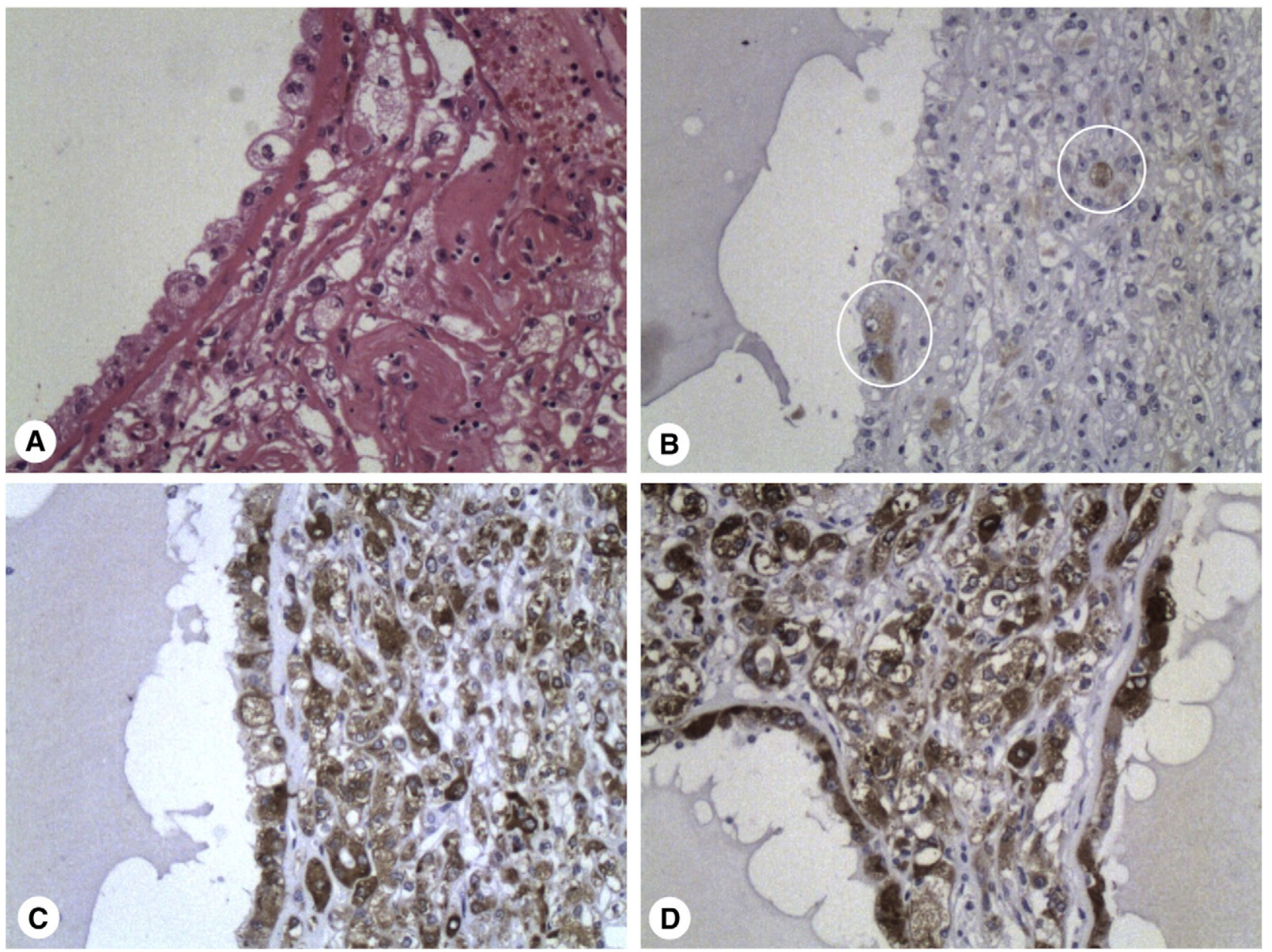

Fig. 4. Microscopic features of the AML with epithelial cysts. (A) Hobnail cell lining (hematoxylin and eosin, magnification $\times 200$ ). (B) Focal expression of HMB45 in tumor cells at the cystic surface (magnification $\times 2400)$. (C and D) Diffuse expression of Melan A in tumor cells at the cystic surface (magnification $\times 400)$. 
cystic AML [5]. Fine and colleagues described the immunophenotype of 4 AML with epithelial cysts highlighting the higher expression of melanocytic markers (HMB45 and Melan A) and of ER, PR, and CD10 in the subepithelial stroma, whereas smooth muscle actin and desmin were less expressed in these zones. The epithelium lining showed strong reactivity to pancytokeratin and focal expression of soy bean agglutinin, a marker of distal nephron epithelium, and no expression of melanocytic markers, ER, PR, CD10, and renal cell carcinoma antigen [6]. A similar pattern of epithelial/subepithelial stroma immunoreactivity was reported in 2 additional cases [4,9]. Those observations led to the hypothesis that (1) subepithelial stroma may represent a dual differentiation in AML into melanocytic and endometrial/müllerian lineages, based on expression of ER, PR, and CD10, mirroring the dual differentiation in smooth muscle cells from AMLs, which shares melanocytic and muscular markers, and (2) the epithelial lining in these cysts is derived from entrapped native collecting duct epithelium [6]. Our observation of focal expression of HMB45 and diffuse and strong expression of Melan A in the epithelial lining of these cysts strongly suggests that those cysts are entirely derived from AML and share with the other neoplastic components the same immunoreactivity. Conversely, this discrepancy may be explained by the fact that the present case is the only case in which the immunoreactivity of epithelial cysts was evaluated in an EAML. To our knowledge, there is a Chinese series of 3 EAML (2 with renal origin and 1 from retroperitoneum) with the description of cystic changes. There was no information on the amount of epithelioid component in this tumors, and no immunohistochemical study focusing the cystic component was performed [10].

In conclusion, we report a rare combination of 2 variants of AML. Both epithelioid and cystic components pose difficulties for the diagnosis to the surgical pathologist. We report for the first time the immunohistochemical study of the cystic component in an EAML. In contrast to the immunoreactivity reported in typical AML, the present case shows obvious expression of melanocytic markers. This is strong evidence that these cysts are neoplastic and derived from AML, rather than entrapped native collecting duct epithelium.

\section{References}

[1] Martignoni G, Amin MB. Angiomyolipoma. In: Eble JN, Sauter G, Epstein JI, Sesterhenn IA, editors. Pathology and genetics of tumours of the urinary system and male genital organs. Lyon: IARC Press; 2004. p. 65-7.

[2] Amin MB. Epithelioid angiomyolipoma. In: Eble JN, Sauter G, Epstein JI, Sesterhenn IA, editors. Pathology and genetics of tumours of the urinary system and male genital organs. Lyon: IARC Press; 2004. p. 68-9.

[3] Faraji H, Nguyen BN, Mai KT. Renal epithelioid angiomyolipoma: a study of six cases and a meta-analytic study. Development of criteria for screening the entity with prognostic significance. Histopathology 2009;55(5):525-34.

[4] Armah HB, Yin M, Rao UN, Parwani AV. Angiomyolipoma with epithelial cysts (AMLEC): a rare but distinct variant of angiomyolipoma. Diagn Pathol 2007;2:11.

[5] Davis CJ, Barton JH, Sesterhenn IA. Cystic angiomyolipoma of the kidney: a clinicopathologic description of 11 cases. Mod Pathol 2006;19(5):669-74.

[6] Fine SW, Reuter VE, Epstein JI, Argani P. Angiomyolipoma with epithelial cysts (AMLEC): a distinct cystic variant of angiomyolipoma. Am J Surg Pathol 2006;30(5):593-9.

[7] Aydin H, Magi-Galluzzi C, Lane BR, Sercia L, Lopez JI, Rini BI, Zhou M. Renal angiomyolipoma: clinicopathologic study of 194 cases with emphasis on the epithelioid histology and tuberous sclerosis association. Am J Surg Pathol 2009;33(2):289-97.

[8] Brimo F, Robinson B, Guo C, Zhou M, Latour M, Epstein JI. Renal epithelioid angiomyolipoma with atypia: a series of 40 cases with emphasis on clinicopathologic prognostic indicators of malignancy. Am J Surg Pathol 2010;34(5):715-22.

[9] Mikami S, Oya M, Mukai M. Angiomyolipoma with epithelial cysts of the kidney in a man. Pathol Int 2008;58(10):664-7.

[10] Hwang HC, Hwang JI, Hung SW, Gueng MK, Yang CS, Chen CCC. Epithelioid angiomyolipoma: an overview of five cases with the concept of PEComa. Chin J Radiol 2008;33:253-60. 